\title{
Efficacy and Safety of a New Protocol for Fast Intubation in Patients With Covid-19 (Sars-Cov-2)
}

\author{
Ricardo Eliud Cisneros Becerra*, Norma G López Cabrera, Janet García González, Gustavo Adolfo Millán \\ Cornejo, Belia Inés Garduño Chávez, Elda Rocio Maltos Tamez, and Dionisio Palacios Ríos
}

Department of Anesthesiology, University Hospital “Dr. José Eleuterio González”, Autonomous University of Nuevo Leon, Mexico

*Corresponding author: Ricardo Eliud Cisneros Becerra, Department of Anesthesiology, University Hospital "Dr. José Eleuterio González", Autonomous University of Nuevo Leon, Mexico.
Received Date: August 17, 2020

Published Date: Septembr 16, 2020

\section{Abstract}

Introduction: Severe COVID-19 illness is characterized by the development of Acute Respiratory Distress Syndrome (ARDS), for which the mainstay of treatment is represented by mechanical ventilation. Mortality associated with ARDS due to other causes is in the range of 40-60\% but currently available data are not yet sufficient to draw safe conclusions on the prognosis of COVID-19 patients who require mechanical ventilation.

Materials and Methods: This is a Cross-sectional and retrospective study in which we want to determine if the new intubation protocol developed in our center is safe and efficient to achieve effective intubation in patients with COVID-19 as well as to avoid the transmission of the virus in anesthesiology specialists.

Results: The average time to achieve an effective intubation was 49 seconds (range: 35-75 seconds). The number of patients in whom it was possible to achieve intubation on the first attempt was 55 (92.45\%). The number of patients who failed to intubate on the first attempt was 4 , corresponding to $7.55 \%$.

Conclusion: The protocol for intubation of patients COVID19 that we used demonstrated effective results at the time of its application, ensuring the safety of the participating health personnel, as well as all the intubation procedures that were carried out in an effective way.

Keywords: COVID-19; Rapid intubation; Personal protective equipment; SARS-CoV-2; Time intubation

\section{Introduction}

The World Health Organization (WHO) and the International Committee on Taxonomy of Viruses recently called the disease, COVID-19, and the virus, severe acute respiratory syndrome coronavirus 2 (SARS-CoV-2) [1]. Severe COVID-19 illness is characterized by the development of Acute Respiratory Distress Syndrome (ARDS), for which the mainstay treatment is mechanical ventilation [2]. As of 2 April 2020, the worldwide pandemic of coronavirus disease has affected more than $1,000,000$ people and taken the lives of more than 50,000 [3].

Cough is one of the major ways of human-to-human viral spread and is one of the prevalent features of this infectious disease; any airway instrumentation can also exacerbate it [4]. Mortality associated with ARDS due to other causes is in the range of 40$60 \%$, but currently available data are not yet sufficient to draw safe conclusions on the prognosis of COVID-19 patients who require mechanical ventilation [2]. Even with optimal supportive care in the intensive care unit (ICU), many patients develop multiorgan failure and die a few days after the initiation of treatment in the ICU [2]. The number of patients requiring tracheal intubation or tracheostomy has increased dramatically in March and April 2020 with the outbreak of COVID-19 [5].

Well-proven medical professionals who will choose whom to intubate should be aware that they are not deciding who will live 
and who will die, but who is more likely to survive [2]. If intubation is necessary in a COVID-19 patient, death may be a more likely outcome than survival even with optimal treatment in the ICU. The course of the disease and the possibility of intubation must be discussed with patients early in the course of their hospitalization (preferably on admission), so they can make an informed decision about the possibility to undergo mechanical ventilation. Given the low chance of recovery and the long-term complications associated with prolonged stays in the ICU, a Do Not Intubate (DNI) order would not be irrational or self-destructive, even among young healthy adults without comorbidities [2].

During the 2003 epidemic of Severe Acute Respiratory Syndrome (SARS) in Toronto, Canada, it was apparent that health care workers were at risk of infection, particularly those involved in airway-related procedures such as endotracheal intubation [1]. Tracheal intubation in patients with COVID-19 creates a risk of physiological compromise in patients and healthcare providers. Expert and management recommendations are urgently needed in these patients [6]. Medical professionals caring for patients with coronavirus disease 2019 are at high risk of contracting the infection [7]. Aerosol generating procedures, such as noninvasive ventilation (NIV), high-flow nasal cannula (HFNC), bag-mask ventilation, and intubation are of particularly high risk [8].

Expert and management recommendations needed urgently in these patients. Because the infection is transmitted through droplets (and possibly aerosols), optimal precautions must be used to prevent the spread of the virus when managing the airway, particularly during intubation and extubation [3]. The first step in its algorithm for tracheal intubation of critically ill adults adapted for COVID-19, which states that medical personal must wear fully verified personal protective equipment (PPE) [9]. Recommended PPE for contact with critically ill patients with confirmed or suspected 2019-nCoV infection includes fluid-resistant gown, gloves, eye protection, full-face shield and fit-tested $\mathrm{N} 95$ respirators.
Hair covers or hoods should also be worn. Longer sleeved gloves are preferred (if available) to prevent exposure of the wrists with glove slippage [10]. We recommend that a specialized expert in the procedure should perform endotracheal intubation, and early intubation should be considered in patients with impaired respiratory status. In all cases, airway backup plans must be ready [8]. Intubation in the COVID-19 era is a learning experience for everyone, and the use of social media to distribute large volumes of knowledge has been recognized [9].

The hypothesis raised is that by performing a protocol for the safe performance of intubation of patients with COVID-19, the incidence of cases within health personnel will decrease. The objective of this study was to establish a safety protocol for the intubation of patients with COVID-19 to make impact decisions for the benefit of our society and health workers.

\section{Materials and methods}

This is a Cross-sectional and retrospective study in which we want to determine if the new intubation protocol developed in our center is safe and efficient to achieve an effective intubation in patients with COVID-19 as well as to avoid the transmission of the virus in anesthesiology specialists.

The necessary materials and the proposed intubation protocol are described below:

This procedure required an expert in airway management dressed in PPE within the intubation area (Figure 1), as well as a second physician trained in airway management dressed in PPE within the intubation area. Likewise, the help of a nurse trained in medication assistance protocols and airways dressed in PPE within the intubation area was also requested, along with the support of inhalation therapy personnel dressed in PPE within the intubation area. We needed a professional verifier for correct placement of the PPE outside the intubation area, as well as tracheostomy surgery equipment outside the intubation area.

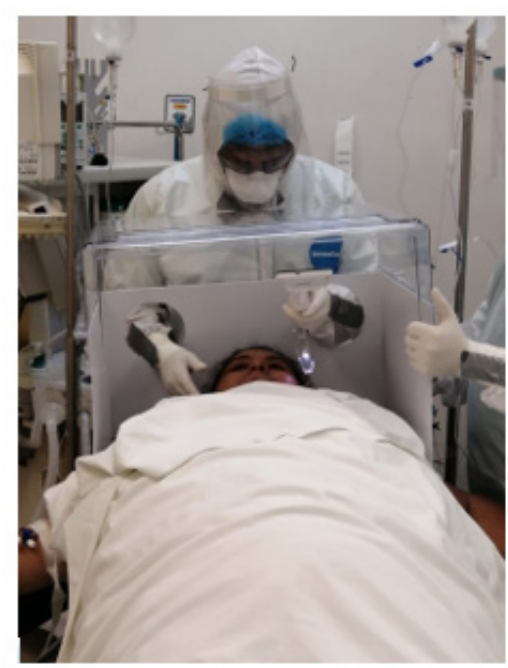

Figure 1: Medical personnel about to perform a procedure inside the intubation area wearing the PPE. 


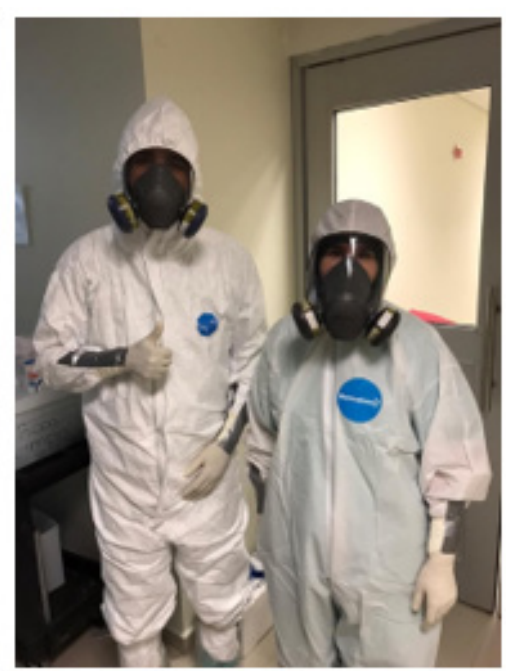

Figure 2: Personnel wearing full PPE to perform procedures safely.

The material needed to comply with this protocol was: Personal Protective Equipment (PPE) (Figure 2), vital signs monitor with capnography, antifog solution, video laryngoscope, laryngoscope with Mac 2, 3 and 4 blades, Miller 1, 2, 3, disposable guides, AMBU face masks ( 3 sizes for adults), balloon orotracheal tubes. Adults (6.5, 7.0, 7.5, 8.0, 8.5), IGel No. 3, 4, and 5 laryngeal masks, closed suction kit firing with Yankauer cannula (or conventional suction system and conventional suction probes), enough folded sheets for pillows for an elevation of $7 \mathrm{~cm}$ to $10 \mathrm{~cm}$ and enough sheets to ramp in case of an obese patient (approximately 10 to 15 sheets), Guedel cannulas, HME antiviral filters at each ventilation interface (ventilation mask, orotracheal tube, ventilator connection), AMBU or BAIN ventilation system, airway cart available with cricothyroidotomy kit, endotracheal tube exchangers, and laryngeal masks outside of the intubation area, as well as intubation medications: midazolam, fentanyl, propofol, ketamine, etomidate, rocuronium, lidocaine, atropine, succinylcholine, nalbuphine, a red car available (must include vasopressors and equipment for intravenous infusion management), enough additional material al: gauze, pads or fields, gloves, adhesive cloth, suction tube, as well as the work area must have an exclusive table or surface to place new clean material and other to place the already used material. All the mentioned material was provided in the intubation area.

The placement of personal protective equipment and the proper disposal and disposal thereof were prioritized to avoid automatic contamination.

Before using the PPE, the medical personnel collected their hair, as well as removed their jewelry, identifications, and empty pockets, adjusted their footwear and glasses, performed hand hygiene, and also checked the availability of personal protective equipment.

The list of personal protective equipment included: Boots, N95 mask, safety glasses, 2 caps, protective mask, ovelrall, scrubs and gloves.
To put on the protective equipment, boots and a surgical cap were first put on, then the first pair of gloves (or internals), diving suits (if there are any in stock), overalls were put on (without putting on a hood), an N95 mouth cover and also glasses or magnifying glasses if necessary), mask or mica was also put on, put on overalls hood, high boots must be worn over overalls, second pair of gloves (or external) was placed on top of overalls sleeves, used gray tape over the second pair of overalls gloves and sleeves, and a surgical gown was also placed over the overalls.

For the removal of personal protective equipment, hand hygiene was first performed with an external glove, with disinfectant solution, then boots were removed, hand hygiene was performed again with external gloves, with disinfectant solution to remove the hat of the overall jumpsuit. back to front, hand hygiene was again performed with external gloves, with disinfectant solution, then the overalls were removed together with external gloves, hand hygiene was again performed with disinfectant solution with 2nd pair of (internal) gloves put on and then remove of the mask / lenses, of the elastic, from back to front, after this hand hygiene was done with internal gloves with disinfectant solution, the mask was removed from back to front, after that perform hand hygiene with internal gloves, with disinfecting solution, remove the surgical cap and redo hand hygiene with internal gloves, with disinfecting solution Before removing the inner gloves and finally, hand hygiene was again performed with disinfectant / soap solution.

The anesthesia induction protocol for intubation indicated that there should be an anesthesiologist assigned for intubation who is considered experienced for it, and was also accompanied to receive support from positive pressure, but in case of severe hypoxia, this should be exercised (manage the high-frequency ventilation with low volume). Speaking about induction drugs that are used, in hemodynamically stable patients we suggest propofol 1.5-2mg per $\mathrm{kg}$ in combination with low doses of some fentanyl-type opioids 1-2mc per kg or nalbuphine (nubain), whereas in hemodynamically 
unstable patients they can be administer low doses of IV midazolam (single dose of 1-3 mg) combined with etomidate (.1-.2 mg per kg) or IV ketamine $2 \mathrm{mg}$ per kg of weight. We suggest avoiding awake intubation in all patients except when there is a history or evidence of a difficult airway, otherwise we recommend deep relaxation use based on rocuronium (1 $\mathrm{mg}$ per $\mathrm{kg}$ ) or succinylcholine at 1-2 mg per kg of weight too slowly to avoid twitching (the latter will be ruled out if the patient has evidence or data suggesting a high risk of hyperkalemia). Rocuronium can be used by allowing a minute of apnea to pass before intubation. Another option may be relaxation with an administered priming dose of 5 to $10 \mathrm{mg}$ of rocuronium before the selected hypnotic and the rest of the dose of the subsequent relaxant, leaving a 1-minute apnea allowed for intubation. We recommend the administration of $1 \mathrm{mg}$ of lidocaine per kg of IV weight in all patients to reduce the cough reflex. In case of using intravenous ketamine, atropine $10 \mathrm{mc}$ should be used for every $10 \mathrm{~kg}$ of weight.

External laryngeal manipulation is valid for better tracheal vision, the use of a guide and / or cricoid depression to avoid gastroesophageal reflux and aspiration.

Intubation was confirmed in the first instance with visualization of elevated lung fields and capnography. It was immediately connected to the fan. Ventilation was not given with Ambu. The tube depth will be $23 \mathrm{~cm}$ and $21 \mathrm{~cm}$ for men and women, respectively. Non-auscultation was considered if possible. Chest radiography is preferred.

We also recommend using high collection filters between each ventilation interface (tube, mask, fan) at the end of the expiratory valve hose or between the tube and the hose of the ventilation system, in case of doubt or failure of intubation. tracheal tube, the tracheal tube is clamped, and the ventilator is disconnected before disconnecting the tube from the rest of the system, subsequently an endotracheal tube guide will be placed in all patients and the integrity of the balloon will be confirmed. The need to previously optimize the hemodynamic state (intramuscular volume and vasopressors) will be considered. As far as possible, the intubation video laryngoscope should be used first.

The sequence that was followed when faced with a difficult airway is: If the patient was unable to intubate the first attempt, he can receive assistance with AMBU and mask management with very low volumes and high frequency until recovery, then the factor was analyzed correctly (pillow, head position, type or size of latoscope slide, tube shape, laryngoscopy technique), a second intubation attempt was also made, if this is unsuccessful, a supraglottic device (laryngeal mask is used or use of stylet), if not successful, a surgical airway was performed.

All anesthesiologists underwent a PCR test to detect SARS-CoV-2 and subsequently one month later a COVID-19 antibody detection test, and how many results were positive were studied, in the same way, the number of intubated patients, the number of attempts made, as well as the clinical and demographic characteristics of the doctors who participated in the procedures were collected.

\section{Results}

Twelve residents of anesthesiology (6 male and 6 female) were included in the present study, who performed the intubation protocol described above as part of the treatment of critically ill patients between February and June 2020. The number of intubated patients was $53(100 \%)$. The average time to achieve an effective intubation was 49 seconds \pm 7.16 (range: $35-75$ seconds). The number of patients in whom it was possible to achieve intubation on the first attempt was 55 (92.45\%). The number of patients who failed to intubate on the first attempt was 4, corresponding to $7.55 \%$. At the end of the study period, PCR tests were performed as well as detection of blood antibodies against coronavirus for all anesthesiologists, resulting in $0 \%$ positive results, in the same way there were no complications during or after the procedures related to the anesthetic method.

\section{Discussion}

Begley et al carried out a study in which 12 experienced anesthesiologists ( 2 women, 10 men) experienced 36 intubations to COVID-19 positive patients without use aerosol box, as well as used early-generation box and latest-generation box. Intubation time with no aerosol box was significantly shorter than with the early-generation box (median (IQR [range]) 42.9 (32.9-46.9 [30.957.6]) s vs. 82.1 (45.1-98.3 [30.8-180.0]) s, $p=0.002)$ and the latestgeneration box (52.4 (43.1-70.3 [35.7-169.2]) s, p=0.008) [11].

No intubations without an aerosol box took more than $1 \mathrm{~min}$, whereas 14/24 (58\%) intubations with a box took over $1 \mathrm{~min}$ and $4 / 24$ (17\%) took over 2 min (including the failure) [11]. In our study, 12 residents ( 6 men, 6 women) intubated a total of 53 patients where effective intubations were achieved in an average time of 49 seconds (range: 35-75 seconds), delaying 6 seconds more than those performed without a box aerosol box than in Yang's study, and achieving less time compared to the other 2 groups already mentioned.

In the study carried out by Begley et al, there was a failed intubation ( $>180 \mathrm{~s}$ ) in the early generation box group [11]. In our study, there were a total of $4(7.55 \%)$ failed interventions where patients could not be intubated on the first attempt.

Begley et al reported that only 2/12 anesthetists (17\%) achieved intubations of less than $60 \mathrm{~s}$ using both boxes. Some anesthetists experienced significantly longer intubation times than others [11].

\section{Conclusion}

The protocol for intubation of patients COVID19 that we used, demonstrated effective results at the time of its application, ensuring the safety of the participating health personnel, as well as all the intubation procedures that were carried out efficiently. 
During the elaborated intubations, it was observed that most of them were made on the first attempt with great effectiveness, while a small part of the intubations were made on the second attempt, this caused by external factors such as the application technique or the condition in the one the patient was in.

\section{Acknowledgement}

None.

\section{Conflict of Interest}

No conflict of interest.

\section{References}

1. Orser BA (2020) Recommendations for Endotracheal Intubation of COVID-19 Patients. Anesth Analg 130(5): 1109-1110.

2. Zareifopopulos N, Lagadinou M, Karela A, Karantzogiannis G, Velissaris D (2020) Intubation and Mechanical Ventilation of Patients With COVID-19: What Should We Tell Them? Monaldi Arch Chest Dis 90(1).

3. Asenjo JF (2020) Safer intubation and extubation of patients with COVID-19. Can J Anaesth 67(9): 1276-1278.

4. Aminnejad R, Salimi A, Saeidi M (2020) Lidocaine during intubation and extubation in patients with coronavirus disease (COVID-19). Can J Anaesth 67(6): 759 .
5. Wali A, Rizzo V, Bille A, Routledge T, Chambers AJ (2020) Pneumomediastinum following intubation in COVID-19 patients: a case series. Anaesthesia 75(8):1076-1081.

6. Yao W, Wang T, Jiang B, Gao F, Wang L, et al. (2020) Emergency tracheal intubation in 202 patients with COVID-19 in Wuhan, China: lessons learnt and international expert recommendations. Br J Anaesth 125(1): e28-e37.

7. Chang D, Xu H, Rebaza A, Sharma L, Dela Cruz CS (2020) Protecting health-care workers from subclinical coronavirus infection. Lancet Respir Med 8(3): e13.

8. Cheung JCH, Ho LT, Cheng JV, Cham EYK, Lam KN (2020) Staff safety during emergency airway management for COVID-19 in Hong Kong. Lancet Respir Med 8(4): e19.

9. Kearsley R (2020) Consensus guidelines for managing the airway in patients with COVID-19. Anaesthesia 75(7).

10. Wax RS, Christian MD (2020) Practical recommendations for critical care and anesthesiology teams caring for novel coronavirus (2019$\mathrm{nCoV}$ ) patients. Can J Anaesth 67(5): 568-576.

11. Begley JL, Lavery KE, Nickson CP, Brewster DJ (2020) The aerosol box for intubation in COVID-19 patients: an in-situ simulation crossover study. Anaesthesia 75(8):1014-1021. 